\title{
Improved Understanding of the Loss-of-Symmetry Phenomenon in the Conventional Kalman Filter
}

\section{H. Verhaegen}

\author{
(NASA-TH-100041) IMPROVED ONDERSTANDIMG OF \\ THE LOSS-OF-S Y GUETRI PHEBOAENON IN THE \\ CONVENTIOALL KALAA FILTER (NASA) $14 \mathrm{p}$ \\ N88-14709 \\ CSCL $12 \mathrm{~A}$ \\ Unclas \\ 0118117

$\begin{array}{ll}\text { Unclas } \\ 63 / 64 & 0118117\end{array}$

December 1987 
NASA Technical Memorandum 100041

\section{Improved Understanding of the Loss-of-Symmetry Phenomenon in the Conventional Kalman Filter}

M. H. Verhaegen, Ames Research Center, Moffett Field, California

December 1987

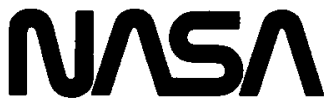

National Aeronautics and

Space Administration

Ames Research Center

Moffett Field, California 94035 


\title{
Improved Understanding of the Loss-of-Symmetry Phenomenon in the Conventional Kalman Filter
}

\author{
M. H. VERHAEGEN
}

\begin{abstract}
This paper corrects an unclear treatment of the conventional Kalman filter implementation as presented by M. H. Verhaegen and P. van Dooren in "Numerical aspects of different Kalman filter implementations," IEEE Trans. Automat. Contr., vol. AC-31, no. 10, pp. 907-917, 1986.
\end{abstract}

This paper shows that habitual, incorrect implementation of the Kalman filter has been the major cause of its sensitivity to the so-called loss-of-symmetry phenomenon.

Keywords

Kalman filter, loss of symmetry, theoretical error analysis

M. H. Verhaegen is an Associate of the US National Research Council, NASA Ames Research Center, Moffett Field, CA-94035 


\section{Introduction}

The loss of symmetry of the state-error-covariance matrix is a well-known phenomenon in the use of the conventional Kalman filter (CKF). This phenomenon has been recognized in experimental (simulation) studies from the origin of the Kalman filter in 1960 [1]. Only recently has a theoretical explanation been given for this phenomenon [2].

From comments received about this paper [2], the given explanation might be subjected to a misinterpretation, which results mainly from the "unclear" specification of what was referred to as the CKF implementation.

In this paper the deficiency is corrected by using the theoretical-error-analysis framework constructed in [2]. Also, the notation introduced in [2] will be retained.

\section{The Conventional Kalman Filter Implementations}

Recall the CKF recursions used in [2]:

$$
\begin{gathered}
R_{k}^{e}=R_{k}+C_{k} P_{k \mid k-1} C_{k}^{\prime} \\
K_{k}=P_{k \mid k-1} C_{k}^{\prime}\left[R_{k}^{e}\right]^{-1} \\
P_{k+1 \mid k}=A_{k}\left[I-P_{k \mid k-1} C_{k}^{\prime}\left[R_{k}^{e}\right]^{-1} C_{k}\right] P_{k \mid k-1} A_{k}^{\prime}+B_{k} Q_{k} B_{k}^{\prime} .
\end{gathered}
$$

The matrix multiplications in the set of recursive relationships (2.1)-(2.3) might be (and have been) executed in various ways. When doing this, it is not even necessary to modify the structure of the relationships as in such altered forms as the so-called (Joseph's) stabilized Kalman filter [3]. Therefore, by focusing on the recursive relationships as given by (2.1)-(2.3), we compare the behavior of two different implementations. A first, and widely used, implementation is represented in Table $\mathrm{I}$ as the CKF1. The second implementation, which is the exact execution of the matrix multiplications, is also represented in Table I as the CKF2. 


\section{TABLE I}

The CKF1 and CKF2 Implementation of the Conventional Kalman Filter

\begin{tabular}{|l|l|l|}
\hline \multicolumn{2}{|c|}{ CKF1 } \\
\hline quantity & \multicolumn{1}{|c|}{ mathematical expression } & number of flops \\
\hline$C P$ & $C_{k} \times P_{k \mid k-1}$ & $n^{2} p$ \\
$R_{k}^{e}$ & $C P \times C_{k}^{\prime}+R_{k}$ & $n p^{2}$ \\
{$\left[R_{k}^{e}\right]^{-1}$} & {$\left[R_{k}^{e}\right]^{-1}$} & $p^{3}$ \\
$K_{k}$ & $C P^{\prime} \times\left[R_{k}^{e}\right]^{-1}$ & $n p^{2}$ \\
$P_{k}^{*}$ & $\left(P_{k \mid k-1}-K_{k} \times C P\right)$ & $n^{2} p$ \\
$P_{k+1 \mid k}$ & $A_{k} \times P_{k}^{*} \times A_{k}^{\prime}+B_{k} \times Q_{k} \times B_{k}^{\prime}$ & $2 n^{3}+n^{2} m+m^{2} n$ \\
\hline
\end{tabular}

\begin{tabular}{|c|c|c|}
\hline \multicolumn{3}{|c|}{ CKF2 } \\
\hline quantity & mathematical expression & number of flops \\
\hline$C P$ & $\star$ & 夫 \\
\hline$R_{k}^{e}$ & $\star$ & $\star$ \\
\hline$\left[R_{k}^{e}\right]^{-1}$ & $\star$ & $\star$ \\
\hline$K_{k}$ & $P_{k \mid k-1} \times C_{k}^{\prime} \times\left[R_{k}^{e}\right]^{-1}$ & $n p^{2}+n^{2} p$ \\
\hline$P_{k}^{\star}$ & $\star$ & $\star$ \\
\hline$P_{k+1 \mid k}$ & $\star$ & - \\
\hline
\end{tabular}

The notation of the $\star$ in Table $I$ indicates that the same mathematical expression is used for the CKF2 implementation as for the CKF1 implementation. This table, therefore, clearly shows that the only minor difference in the two implementations is in the calculation of the Kalman gain, $K_{k}$. The CKF1 in this step exploits the symmetry of $P_{k \mid k-1}$, thereby decreasing the computational complexity. However, it will be shown in the next subsection that it is precisely this fact that makes the implementation especially sensitive to the loss of symmetry.

\section{Theoretical Error Analysis}

In this section the same theoretical-error-analysis framework defined in [2] is used to analyze the propagation of errors through the implementations CKF1 and CKF2, described in Section II. 
Therefore, consider again the representation of the error $\delta P_{k \mid k-1}$ on the state-errorcovariance matrix $P_{k \mid k-1}$ :

$$
\bar{P}_{k \mid k-1}=P_{k \mid k-1}+\delta P_{k \mid k-1}
$$

This single error then builds up through the recursive relationships of the CKF1 and CKF2 implementation as represented in Table II [2]. Here, infinite precision of the computations was assumed. 
TABLE $\mathbf{n}$

Propagation of a Single Error $\delta P_{k \mid k-1}$ Through the CKF1 and CKF2 Implementation of the Conventional Kalman Filter, Using Exact Arithmetic

\begin{tabular}{|c|c|}
\hline \multicolumn{2}{|r|}{ CKF1 } \\
\hline quantity & error \\
\hline$C_{k} \cdot \bar{P}_{k \mid k-1}$ & $C_{k} \delta P_{k \mid k-1}$ \\
\hline$\overline{\left[R_{k}^{e}\right]^{-1}}$ & $-\left[R_{k}^{e}\right]^{-1} C_{k} \delta P_{k \mid k-1} C_{k}^{\prime}\left[R_{k}^{e}\right]^{-1}+O\left(\delta^{2}\right)$ \\
\hline $\bar{K}_{k}$ & $\begin{array}{l}-P_{k \mid k-1}^{\prime} C_{k}^{\prime}\left[R_{k}^{e}\right]^{-1} C_{k} \delta P_{k \mid k-1} C_{k}^{\prime}\left[R_{k}^{e}\right]^{-1}+ \\
\delta P_{k \mid k-1}^{\prime} C_{k}^{\prime}\left[R_{k}^{e}\right]^{-1}+O\left(\delta^{2}\right)\end{array}$ \\
\hline$\overline{P_{k}^{*}}$ & $\delta P_{k \mid k-1}-P_{k \mid k-1}^{\prime} C_{k}^{\prime}\left[R_{k}^{e}\right]^{-1} C_{k} \delta P_{k \mid k-1}+$ \\
\hline & $\begin{array}{l}\left.P_{k \mid k-1}^{\prime} C_{k}^{\prime}\left[R_{k}^{e}\right]^{-1} C_{k} \delta P_{k \mid k-1} C_{k}^{\prime} \mid R_{k}^{e}\right]^{-1} C_{k} P_{k \mid k-1}- \\
\delta P_{k \mid k-1}^{\prime} C_{k}^{\prime}\left[R_{k}^{e}\right]^{-1} C_{k} P_{k \mid k-1}+O\left(\delta^{2}\right)\end{array}$ \\
\hline $\bar{P}_{k+1 \mid k}$ & $A_{k}\left(\delta P_{k \mid k-1}-P_{k \mid k-1}^{\prime} C_{k}^{\prime}\left[R_{k}^{e}\right]^{-1} C_{k} \delta P_{k \mid k-1}+\right.$ \\
\hline & $P_{k \mid k-1}^{\prime} C_{k}^{\prime}\left[R_{k}^{e}\right]^{-1} C_{k} \delta P_{k \mid k-1} C_{k}^{\prime}\left[R_{k}^{e}\right]^{-1} C_{k} P_{k \mid k-1}-$ \\
\hline & $\left.\delta P_{k \mid k-1}^{\prime} C_{k}^{\prime}\left[R_{k}^{e}\right]^{-1} C_{k} P_{k \mid k-1}\right) A_{k}^{\prime}+O\left(\delta^{2}\right)$ \\
\hline \multicolumn{2}{|r|}{ CKF2 } \\
\hline quantity & error \\
\hline$C_{k} \cdot \bar{P}_{k \mid k-1}$ & $C_{k} \delta P_{k \mid k-1}$ \\
\hline$\overline{\left[R_{k}^{e}\right]^{-1}}$ & $-\left[R_{k}^{e}\right]^{-1} C_{k} \delta P_{k \mid k-1} C_{k}^{\prime}\left[R_{k}^{e}\right]^{-1}+O\left(\delta^{2}\right)$ \\
\hline \multirow[t]{2}{*}{$\bar{K}_{k}$} & $-P_{k \mid k-1} C_{k}^{\prime}\left[R_{k}^{e}\right]^{-1} C_{k} \delta P_{k \mid k-1} C_{k}^{\prime}\left[R_{k}^{e}\right]^{-1}+$ \\
\hline & $\delta P_{k \mid k-1} C_{k}^{\prime}\left[R_{k}^{e}\right]^{-1}+O\left(\delta^{2}\right)$ \\
\hline \multirow[t]{3}{*}{$\overline{P_{k}^{*}}$} & $\delta P_{k \mid k-1}-P_{k \mid k-1} C_{k}^{\prime}\left[R_{k}^{e}\right]^{-1} C_{k} \delta P_{k \mid k-1}+$ \\
\hline & $P_{k \mid k-1} C_{k}^{\prime}\left[R_{k}^{e}\right]^{-1} C_{k} \delta P_{k \mid k-1} C_{k}^{\prime}\left[R_{k}^{e}\right]^{-1} C_{k} P_{k \mid k-1}-$ \\
\hline & $\delta P_{k \mid k-1} C_{k}^{\prime}\left[R_{k}^{e}\right]^{-1} C_{k} P_{k \mid k-1}+O\left(\delta^{2}\right)$ \\
\hline \multirow[t]{3}{*}{$\bar{P}_{k+1 \mid k}$} & $A_{k}\left(\delta P_{k \mid k-1}-P_{k \mid k-1} C_{k}^{\prime}\left[R_{k}^{e}\right]^{-1} C_{k} \delta P_{k \mid k-1}+\right.$ \\
\hline & $\left.P_{k \mid k-1} C_{k}^{\prime}\left[R_{k}^{e}\right]^{-1} C_{k} \delta P_{k \mid k-1} C_{k}^{\prime} \mid R_{k}^{e}\right]^{-1} C_{k} P_{k \mid k-1}$ \\
\hline & $\left.\delta P_{k \mid k-1} C_{k}^{\prime}\left[R_{k}^{e}\right]^{-1} C_{k} P_{k \mid k-1}\right) A_{k}^{\prime}+O\left(\delta^{2}\right)$ \\
\hline
\end{tabular}


Summarizing, the error propagation model for the CKF1 becomes:

$$
\delta P_{k+1 \mid k}=F_{k} \delta P_{k \mid k-1} F_{k}^{\prime}+A_{k}\left(\delta P_{k \mid k-1}-\delta P_{k \mid k-1}^{\prime}\right) C_{k}^{\prime} K_{k}^{\prime}+O\left(\delta^{2}\right)
$$

while the model for the CKF2 is:

$$
\delta P_{k+1 \mid k}=F_{k} \delta P_{k \mid k-1} F_{k}^{\prime}+O\left(\delta^{2}\right)
$$

In the equations 3.2 and $3.3, F_{k}$ is the Kalman filter transition matrix $\left(A_{k}-A_{k} K_{k} C_{k}\right)$. The error propagation model (3.2) was also obtained in [2]. However, it is now clear to which of the CKF implementations it corresponds.

The conclusion from this analysis is that an asymmetric error on the state-errorcovariance matrix in the CKF 1 implementation leads to divergence due to the loss of symmetry when the original transition matrix $A_{k}$ is unstable. This phenomenon is a consequence of the implementation, and occurs irrespective of the machine precision. However, when one uses the almost identical implementation of the CKF2, such asymmetric error vanishes when the Kalman filter (KF) has converged.

This analysis clearly shows that the slightly more economical and error-prone implementation of the recursive relationships (2.1)-(2.3), as represented by the CKF1 in Table I, is responsible for the loss-of-symmetry phenomenon, which has been so blindly associated with the CKF in general.

In the next section, the above conclusion is verified in an experimental simulation study.

\section{Experimental Evaluation}

The system representation taken in this experimental analysis is the one used in test 1 of [2]. The attribute of this (time invariant) system model, which is important for the round-off propagation behavior of the two CKF implementations under consideration, is the location of all the eigenvalues of the system transition matrix $A$ on the unit circle. 
Following the assumption as in [2], the single-precision computed quantities, denoted by $\overline{(.)}$, are the erroneous quantities, and the double-precision ones are considered to be errorfree. In this way, the errors on the quantities in the KF recursion are approximated by the difference between their single-precision and their double-precision updates.

In order to verify the conclusion made in the previous section, both the CKF1 and CKF2 implementations are executed for the first 100 recursions in single-precision, and from then on, continue in (double-precision) exact arithmetic. The resulting quantities updated in the Kalman recursion, as denoted in Table I, are then compared to the same quantities updated in exact arithmetic from the first recursion on. The (Frobenius) norm of the differences of these quantities is plotted in Fig. 1 for the CKF1 and in Fig. 2 for the CKF2. These figures obviously demonstrate the conclusion made in Section III.

\section{Concluding Remarks}

The purpose of the analysis in this paper was to clarify a possible misinterpretation of a theoretical explanation given in [2] of the loss-of-symmetry phenomenon of the CKF. The analysis has clearly demonstrated that an "error-prone" way of implementing the CKF has been responsible for the conviction that this filter is the inherent cause of the well-known loss-of-symmetry phenomenon.

Furthermore, the power of the outlined theoretical-error-analysis framework in [2] to analyze the robustness of (Kalman filter) algorithms is again demonstrated. 


\section{References}

[1] R. E. Kalman, "A new approach to linear filtering and prediction problems," Trans. ASME. (J. Basic Eng.), vol. 82D, pp. 34-45, Mar. 1960.

[2] M. H. Verhaegen and P. Van Dooren, "Numerical aspects of different Kalman filter implementations,” IEEE Trans. Automat. Contr., vol. AC-31, no. 10, pp. 907-917, 1986.

[3] B. D. O. Anderson and J. B. Moore, Optimal Filtering. Englewood Cliffs, New Jersey: Prentice Hall, Information and System Sciences Series, 1979. 


\section{Figure Captions}

Fig. 1. Propagation of round-off errors in the CKF1 implementation.

Fig. 2. Propagation of round-off errors in the CKF2 implementation. 


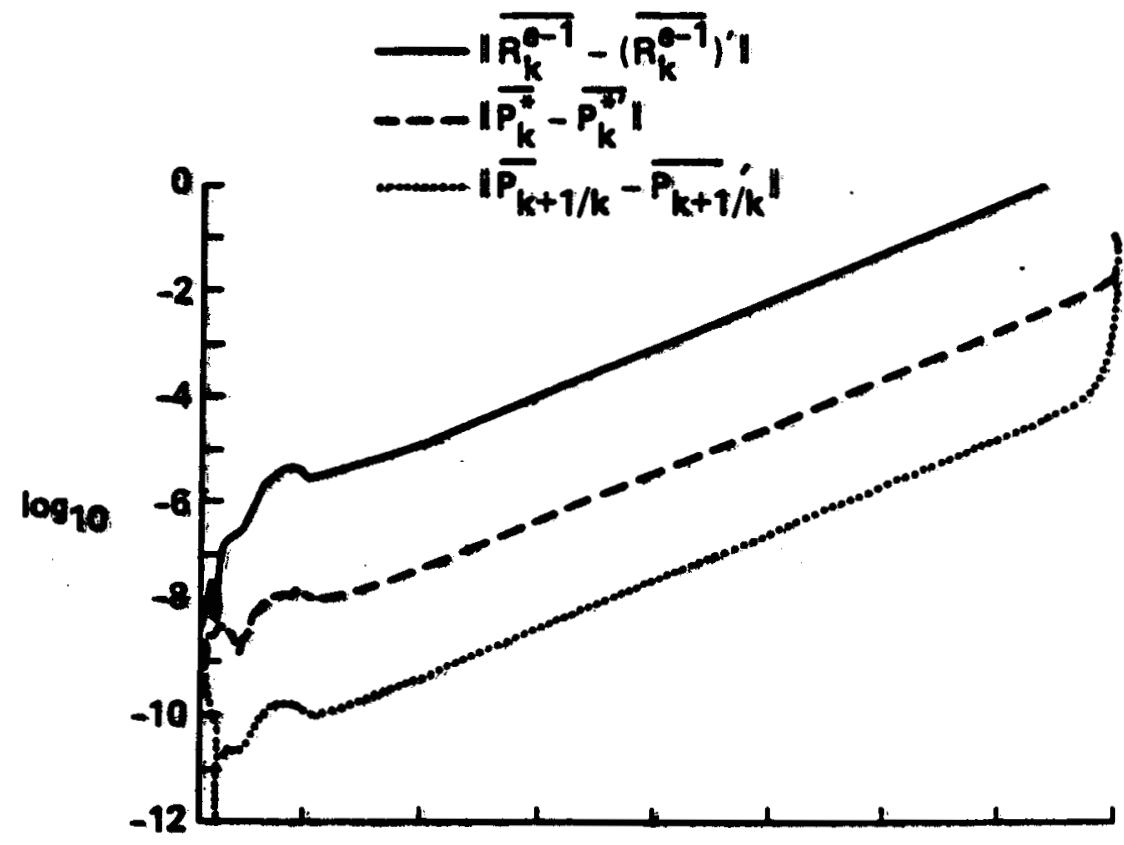

(a) THE LOSS OF STMMETRY IN THE MATRICES $R_{k}^{\circ-1}, P_{k}^{*}$ AND $P_{k+1 / k}$ OF THE CKF1 IMPLEMENTATION

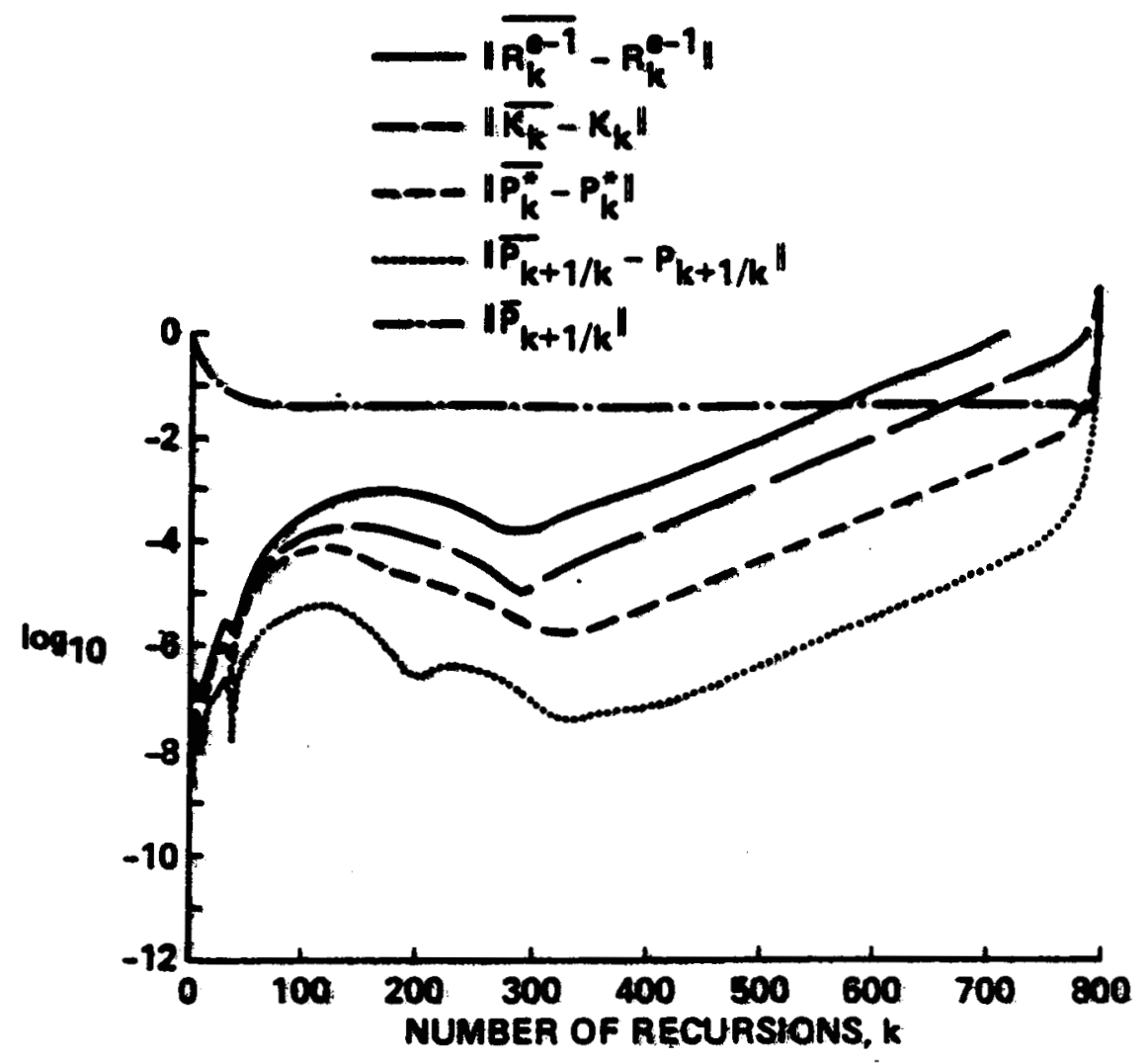

(b) ERRORS ON $R_{k}^{-1}, K_{k}, P_{k}^{*}$ AND $P_{k+1 / k}$ OF THE CKF1 IMPEEMENTATION

Fig. 1 


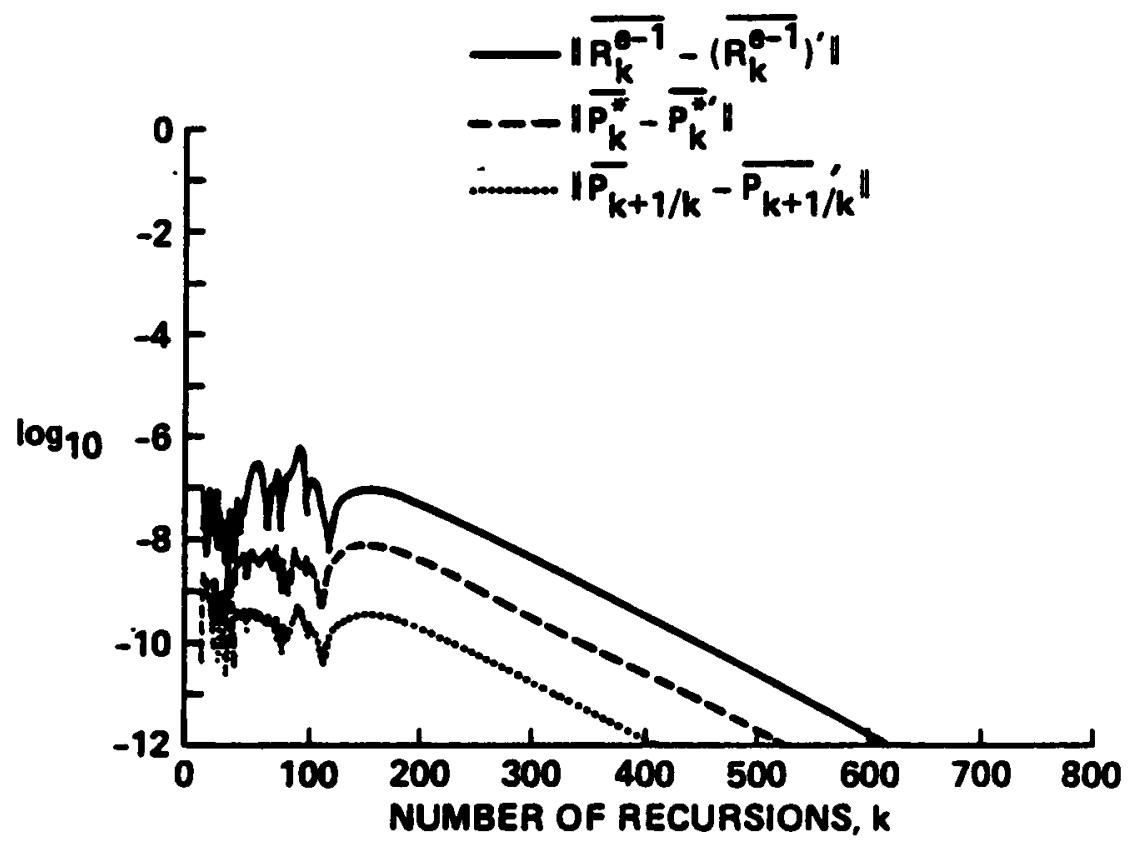

(a) THE LOSS OF SYMMETRY IN THE MATRICES $R_{k}^{6-1}, P_{k}^{*}$ AND $P_{k+1 / k}$ OF THE CKF2 IMPLEMENTATION

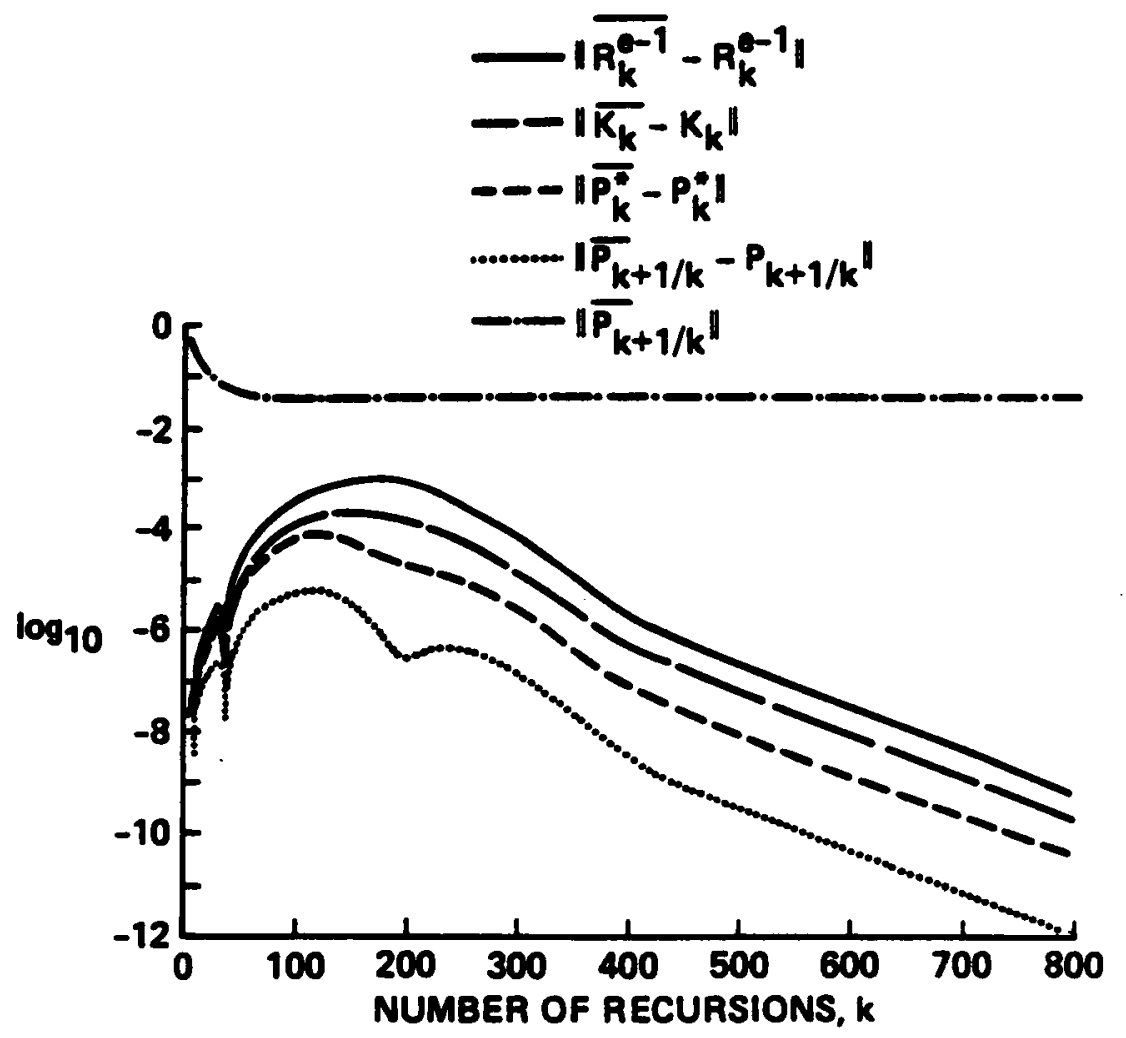

(b) ERRORS ON $R_{k}^{0-1}, K_{k}, P_{k}^{*}$ AND $P_{k+1 / k}$ OF THE CKF2 IMPLEMENTATION

Fig. 2 


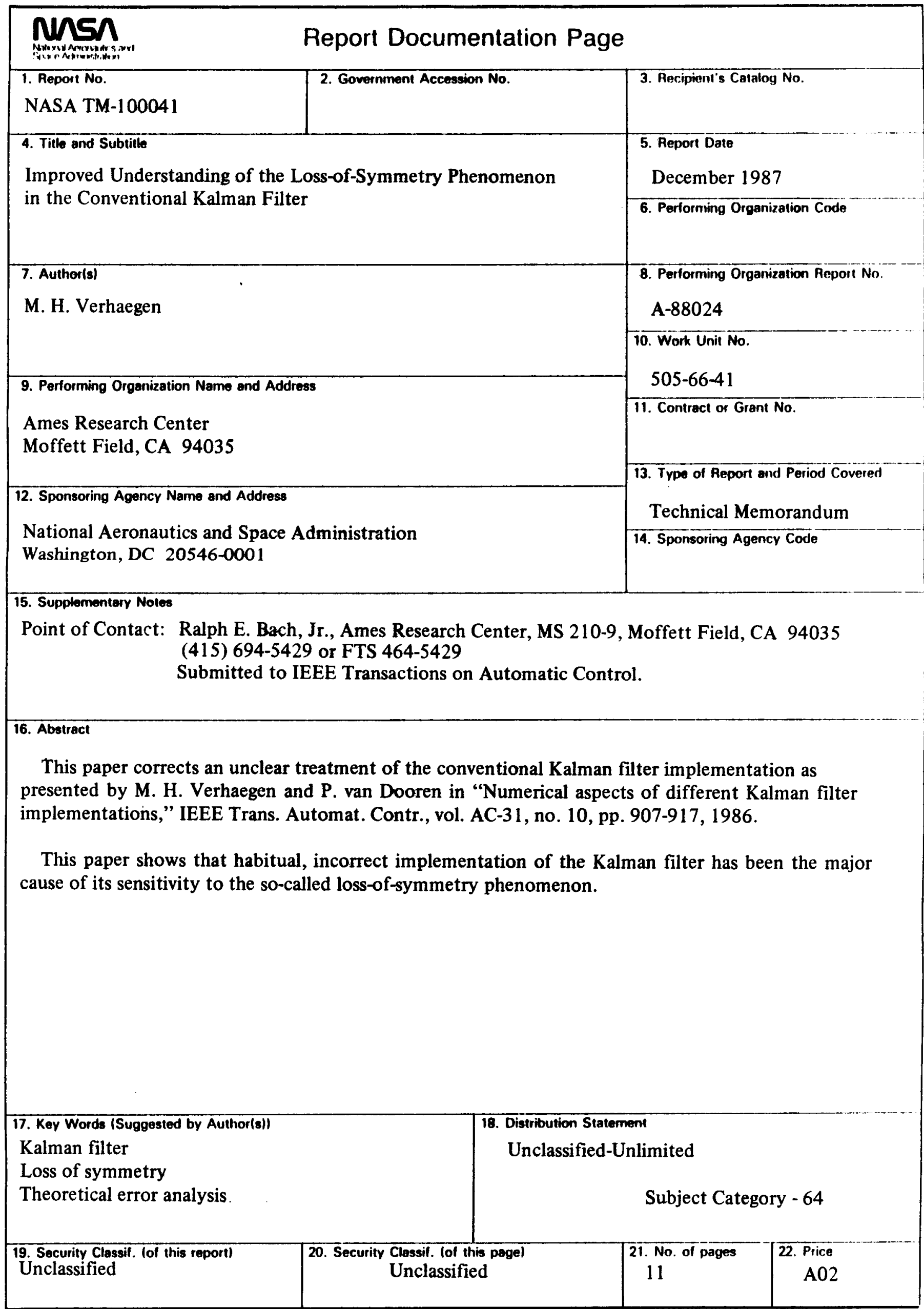

